a study has been made of the potential fields in the neighborhoods of gaseous ions, with particular emphasis on the effects due to adjacent ions.

Methods used heretofore have assumed that each ion is enclosed in a spherical volume of radius $a_{Z},{ }^{1}$ wherein no other ions are permitted. This is, of course, only a first approximation. Using a generalized Thomas-Fermi method it has been found that, for pure iron at i 1.6 million degrees and with three bound electrons, the density of neighboring ions at a distance of $\frac{1}{2} a_{Z}$ from any particular ion is still appreciable. For a gas mixture of the proportions suggested by Harrison Brown ${ }^{2}$ the densities of $\mathrm{H}, \mathrm{He}, \mathrm{O}$ and $F e$ ions at $\frac{1}{2} a_{Z}$ are $76,57,13$ and o per cent of the respective average densities. The accompanying depression of the ionization potentials is only 60 per cent of that computed by Morse's method.

I. Marshak, Morse and York, $A p$. J. III, 2 I4, 1950. 2. Rev. Mod. Phys. 21, 625, Table III, I949.

Perkins Observatory, Ohio Wesleyan and Ohio State Universities, Delaware, Ohio, and Argonne National Laboratory,

\section{King, Ivan. The period-luminosity curve in the Small Magellanic Cloud.}

The existing determination of the periodluminosity curve in the Small Magellanic Cloud depends upon the magnitude standards of the Harvard master sequence in the cloud. Photoelectric measurements have been made of the magnitudes of these sequence stars, in order to strengthen the photometric basis of the curve. Because no smaller focal-plane diaphragm than $2^{\prime \prime}$ was available in the photometer used, the limiting source of error in the magnitude of a sequence star is the contribution of faint neighboring stars to the light measured. Photographic magnitudes of all such faint stars were measured, down to magnitude 18.5 on blue-sensitive and to I7.2 on red-sensitive plates taken with the 6o-inch Rockefeller reflector; and all photometer deflections were corrected for the light contributed by faint stars.

Since the magnitudes of the Cepheid variables were measured in the past on photographs made with the 24-inch Bruce doublet, it was also necessary to determine the color equation of the Bruce. The sequence stars were corrected for this color equation, in order to reduce them to the color system of the Bruce; and the resulting magnitudes were compared with the previously adopted standard magnitudes. The photometric correction deduced was combined with the correction required to reduce Bruce magnitudes of the Cepheids to the international system. The resulting correction to the period-luminosity curve tends to increase the slope of the bright end of the curve; corrections to the faint end are less certain because of increasing scatter in the photoelectric magnitudes of the fainter stars.

Harvard College Observatory, Cambridge, Mass.

Layzer, David. Patterns of term structure in simple spectra.

Theoretically, a pure configuration of electrons outside closed subshells has a definite, calculable pattern of term structure. For example, the two intervals between the three terms of (closed subshells) $\left(p^{2}\right)$ have the theoretical ratio I.5.

The observed patterns, however, often vary widely from atom to atom. For example, the above-mentioned ratio for $\left(I s^{2}\right)\left(2 p^{2}\right)$ is about -5 in $B e \mathrm{I}$ and +8 in $B I$ I.

Discrepancies of this kind are generally, and in my opinion correctly, ascribed to the mixing of configurations. Two obstacles have stood in the way of a successful quantitative theoretical description of configuration mixing: the lack of reliable criteria for recognizing the important components in a configuration mixture; and the lack of workable mathematical machinery for calculating the degree of mixing of given configurations. In this paper I try to overcome both these obstacles.

My first point is that the data can be grouped so as to exhibit useful regularities in the structure of configuration-sequences. A configuration-sequence is defined as a set of identical configurations in an iso-electronic sequence, e.g., $\left(\mathrm{I} s^{2}\right)$ $\left(2 p^{2}\right)$ in BeI, BII, CIII, .... If $\mathrm{I}(Z)$ is the interval between two terms of a configuration in such a sequence, $Z$ denoting the atomic number, then the interval-difference $\mathrm{I}(Z+\mathrm{I})-\mathrm{I}(Z)$ becomes sensibly constant for moderately large $Z$. The set of all independent interval-differences for a given sequence characterizes the sequence as a whole.

A simple theoretical argument now shows that the structure of this set is influenced only by a very special kind of configuration mixing, which I call stable mixing. Only a small number of configurations can participate in a stable mixture, and these may be readily enumerated in every instance. I have found a method of predicting 